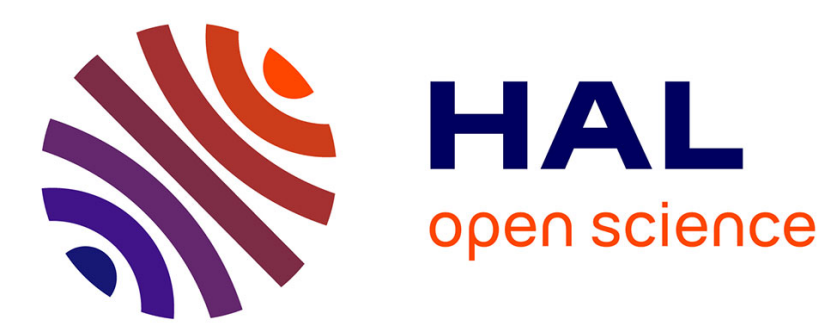

\title{
Hong Kong et le delta de la rivière des Perles : liens économiques et activisme social \\ Chloé Froissart, Yi Xu
}

\section{To cite this version:}

Chloé Froissart, Yi Xu. Hong Kong et le delta de la rivière des Perles : liens économiques et activisme social. Critique, 2014. hal-03168622

\section{HAL Id: hal-03168622 \\ https://hal.science/hal-03168622}

Submitted on 13 Mar 2021

HAL is a multi-disciplinary open access archive for the deposit and dissemination of scientific research documents, whether they are published or not. The documents may come from teaching and research institutions in France or abroad, or from public or private research centers.
L'archive ouverte pluridisciplinaire HAL, est destinée au dépôt et à la diffusion de documents scientifiques de niveau recherche, publiés ou non, émanant des établissements d'enseignement et de recherche français ou étrangers, des laboratoires publics ou privés. 


\section{Hong Kong et le delta de la rivière des Perles: liens économiques et activisme social}

Hong Kong, centre de commerce prospère et intermédiaire entre la Chine et les investissements étrangers, joue depuis longtemps un rôle important dans le développedepuis longtemps un role ment économique du continent ${ }^{1}$. Les échanges et renforcés depuis le lancement des réformes en Chine en 1978, notamdepuis le lancement des reccords du CEPA (Closer Economent en 2003 grâce aux accords du CEPA (Closer Economic Partnership Arrangement) qui lèvent les obstacles au commerce et à l'investissement de part et d'autre de la frontière, permettant aux entreprises hongkongaises de faire plus facilement des affaires en Chine. Ces accords ont également faciemen un important dans l'augmentation des flux de popujoué un rôle important dant désormais se rendre à Hong Kong lation, les Chinois alors qu'ils étaient contraints jusqu'alors de à titre individuel alors qu'ils étaient contraints jusqu'ayages. faire partie de tours organisés par des agences de voyages.

Hong Kong possède dex nationales, des entrepreneurs qualifiés, une expertise technique et de commercialisation reconnue au niveaú mondial, et un secteur des services prospère. De son côté, la Chine
dispose d'importantes ressources foncières et d'une maind'œuvre jeune et presque infiniment extensible. Compte tenu de cette complémentarité, les liens économiques entre la Chine et Hong Kong n'ont cessé de se développer depuis la Chine et Hong Kong nont spéciale (RAS) les réformes, et la Région administrative spéciale (RAS) a été le premier investisseur étranger en Chine au cours des trois dernières décennies, majoritairement dans le delta de

1. D. R. Meyer, Hong Kong as a Global Metropolis, Cambridge (U. K.), Cambridge University Press, 2000, p. 1-4. la rivière des Perles ${ }^{2}$ situé dans la province limitrophe du Guangdong, avec laquelle Hong Kong partage une langue et une culture communes.

Shenzhen, à l'origine petit village de pêcheurs érigé en "Zone économique spéciale » en 1979, aujourd'hui l'une des villes les plus riches de Chine comptant plus de 18 millions d'habitants ${ }^{3}$, est séparée de Hong Kong par une rivière - la rivière Shenzhen - qui peut être franchie en cinq minutes à pied. Chaque jour, plusieurs centaines de milliers de personnes traversent la frontière pour vaquer à leurs occupations professionnelles, faire du tourisme et du shopping ou rejoindre leur lieu d'habitation. Le renforcement de la coopération commerciale s'est donc accompagné d'une rapide intégration géographique, encouragée par un plan d'intégration du « grand delta de la rivière des Perles », promulgué conjointement par les gouvernements de Hong Kong, Macao et du Guangdong en 2009. Ce plan, devant permettre aux résidents de la zone de se déplacer d'un bout à l'autre de la région en moins d'une heure, renforce également la division des activités, Hong Kong étant confirmé dans son rôle de centre financier, de services et de vente au détail.

Malgré ces coopérations et ces complémentarités, la frontière reste une démarcation importante. Hong Kong, État de droit où les libertés d'expression, de réunion, d'organisation, de manifestation sont garanties et où il existe une presse et une justice indépendantes, est traditionnellement le lieu de refuge des Chinois du continent ayant fui les dictatures du Guomindang et du Parti communiste. La garantie des libertés civiles et politiques a constitué un terreau favorable au développement d'un activisme en faveur 'du respect des

2. La région du delta de la rivière des Perles comprend neuf préfectures : Canton, Shenzhen, Zhuhai, Dongguan, Zhongshan, Foshan, Huizhou, Jiangmen, Zhaoqing, situées dans le province du Guangdong.

3. «Zongrenkou niannian baozhang shuomin Shenzhen chengsh jingzheng de meili qiangda » [ "La population totale augmente chaque année révélant le pouvoir d'attraction croissant de la ville de Shenzhen »], http://baoliao.oeeee.com/153180.html ; « Diaocha cheng Shenzhen liudong renkou chao 1500 wan wei huji renkou wubei » [ "Une enquête montre que la population migrante de Shenzhen dépasse 15 millions soit cinq fois plus que la population locale »], http://www.chinanews.com/ gn/2013/10-29/5434760.shtml. 
droits civiques, économiques et sociaux de l'autre côté de la frontière.

Si la perméabilité de la frontière joue un rôle d'intégration économique, elle offre donc en même temps la possibilité de la contestation : il est tout aussi aisé aux militants qu'aux investisseurs de la franchir, et si les seconds créent souvent des situations d'exploitation et contribuent aux problèmes environnementaux de la Chine, Hong Kong fournit aux activistes les conditions pour les dénoncer. Aussi le delta de la rivière des Perles est-il devenu la région de Chine où la contestation sociale est la plus forte et la plus sophistiquée ; même si, paradoxalement, les avancées juridiques ainsi impulsées ont aussi été contrecarrées par les entrepreneurs hongkongais.

Le développement des liens économiques et leur coût humain

La politique de réforme et d'ouverture qui, à partir de 1978, permet aux capitaux étrangers d'entrer en Chine, et favorise d'emblée la province du Guangdong - puisque trois de ses villes: Shenzhen, Zhuhai et Shantou, acquièrent en 1980 le statut de «zones économiques spéciales ${ }^{4}$ »-, a coïncidé avec la restructuration économique de Hong Kong. Dans les années 1980, les entreprises du territoire sont confrontées à une augmentation des prix du foncier et de la main-d'œuvre, ainsi qu'à la concurrence de la Malaisie, de l'Indonésie et de la Thailande, également spécialisées dans des industries intensives en travail. Elles profitent alors du peu de réglementation en matière de travail et d'environnement en Chine, qui permet des coûts de production extrêmement bas, ainsi que de l'abondance des terrains plats, pour délocaliser massivement leurs usines dans le delta ${ }^{5}$. Selon les chiffres du gouvernement de Hong Kong, 94 \% des délocalisations entre 1989 et 1992 ont été effectuées vers le Guangdong, dont $43 \%$ à Shenzhen et $17 \%$ à Foshan ${ }^{6}$. Mais la gestion et le contrôle des entreprises, ainsi que les opérations à plus forte valeur

4. La quatrième zone économique spéciale est Xiamen, dans la province du Fujian.

5. F. Xiaoyun (éd.), Xianggang gongye 2000 [Hong Kong Industries

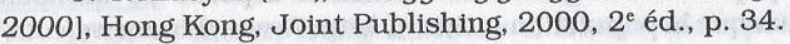

6. Ibid., p. 36. ajoutée, ont été largement maintenus à Hong Kong ${ }^{7}$.

Dans les années 1980 et 1990, la Chine est le pays en voie de développement qui attire le plus d'investissements étrangers au monde ${ }^{8}$; en 2003 , elle dépasse les États-Unis pour devenir le premier pays attirant les investissements directs étrangers. D'emblée, les capitaux hongkongais on prédominé et leur part n'a cessé d'augmenter au cours des dernières années, comme le montre le tableau suivant:

Les cinq premiers pays / régions en termes d'investissements directs étrangers

Années Cinq premiers pays / régions

(calculé en fonction de la valeur réelle des capitaux étrangers utilisés en milliards de $\$$ américains)

\begin{tabular}{|c|c|c|c|c|c|}
\hline 2013 & $\begin{array}{l}\text { Hong Kong } \\
(78.3)\end{array}$ & $\begin{array}{l}\text { Singapour } \\
(7.33)\end{array}$ & $\begin{array}{l}\text { Japon } \\
(7.06)\end{array}$ & $\begin{array}{l}\text { Taiwan } \\
(5.25)\end{array}$ & $\begin{array}{l}\text { EU } \\
(3.35)\end{array}$ \\
\hline 2012 & $\begin{array}{l}\text { Hong Kong } \\
(71.29)\end{array}$ & \begin{tabular}{|l|} 
Japon \\
$(7.38)$
\end{tabular} & $\begin{array}{l}\text { Singapour } \\
(6.54)\end{array}$ & $\begin{array}{l}\text { Taiwan } \\
(6.18)\end{array}$ & $\begin{array}{l}\text { EU } \\
(3.13)\end{array}$ \\
\hline 2011 & $\begin{array}{l}\text { Hong Kong } \\
(77.01)\end{array}$ & $\begin{array}{l}\text { Taiwan } \\
(6.73)\end{array}$ & \begin{tabular}{|l|} 
Japon \\
$(6.35)$ \\
\end{tabular} & $\begin{array}{l}\text { Singapour } \\
(6.33)\end{array}$ & $\begin{array}{l}\mathrm{EU} \\
(2.99)\end{array}$ \\
\hline 2010 & $\begin{array}{l}\text { Hong Kong } \\
\text { (67.47) }\end{array}$ & $\begin{array}{l}\text { Taiwan } \\
(6.7)\end{array}$ & $\begin{array}{l}\text { Singapour } \\
(5.66)\end{array}$ & $\begin{array}{l}\text { Japon } \\
(4.24)\end{array}$ & \begin{tabular}{|l|} 
EU \\
$(4.05)$ \\
\end{tabular} \\
\hline 2009 & $\begin{array}{l}\text { Hong Kong } \\
\text { (53.99) }\end{array}$ & $\begin{array}{l}\text { Taiwan } \\
(6.56)\end{array}$ & $\begin{array}{l}\text { Japon } \\
(4.12)\end{array}$ & $\begin{array}{l}\text { Singapour } \\
(3.89)\end{array}$ & \begin{tabular}{|l|}
$\mathrm{EU}$ \\
$(3.58)$ \\
\end{tabular} \\
\hline 2008 & $\begin{array}{l}\text { Hong Kong } \\
(41.04)\end{array}$ & $\begin{array}{l}\text { îles Vierges } \\
\text { britanniques } \\
(15.95)\end{array}$ & $\begin{array}{l}\text { Singapour } \\
(4.44)\end{array}$ & $\begin{array}{l}\text { Japon } \\
\text { (3.65) }\end{array}$ & $\begin{array}{l}\text { Îles } \\
\text { Caïmans } \\
(3.15) \\
\end{array}$ \\
\hline 2007 & $\begin{array}{l}\text { Hong Kong } \\
(27.7)\end{array}$ & $\begin{array}{l}\text { Îles Vierges } \\
\text { britanniques } \\
(16.55)\end{array}$ & $\begin{array}{l}\text { Corée du } \\
\text { Sud (3.67) }\end{array}$ & $\begin{array}{l}\text { Japon } \\
\text { (3.59) }\end{array}$ & $\begin{array}{l}\text { Singapour } \\
(3.18)\end{array}$ \\
\hline 2006 & $\begin{array}{l}\text { Hong Kong } \\
\text { (21.31) }\end{array}$ & $\begin{array}{l}\text { Îles Vierges } \\
\text { britanniques } \\
\text { (11.68) }\end{array}$ & $\begin{array}{l}\text { Japon } \\
(4.76)\end{array}$ & $\begin{array}{l}\text { Corée du } \\
\text { Sud (3.99) }\end{array}$ & $\begin{array}{l}\mathrm{EU} \\
(2.99)\end{array}$ \\
\hline
\end{tabular}

Source : ministère du Commerce chinois.

7. Th. P. Rohlen, Hong Kong and the Pearl River Delta : "One Country, Two Systems " in the Emerging Metropolitan Context. Stanford, Stanford University Press, 2000.

8. M. E. Gallagher, Contagious Capitalism. Globalization and the Politics of Labor in China, Princeton, Princeton University Press, 2005, p. 1-2. 
En 1995, les entrepreneurs hongkongais détenaient plus de 20000 usines employant environ 4,5 à 5 millions de travailleurs dans le delta de la rivière des Perles ${ }^{9}$. En 2006, il y avait 57500 usines hongkongaises dans le delta employant plus de 10 millions d'ouvriers ${ }^{10}$. Spécialisées dans la manufacture (textile, confection, chaussures, jouets puis électronique) et orientées majoritairement vers l'exportation, les usines hongkongaises du delta sont essentiellement des sous-traitants de grandes multinationales. Situées au plus sous-tiale, elles sont connues mondiale, elles sont connues - avec les entreprises taiwanaises - pour présenter les plus mauvaises conditions de travail et les plus bas salaires, comparées aux entreprises européennes ou américaines.

Ces usines se nourrissent d'une main-d'œuvre flexible et bon marché venue de la campagne qui, n'étant pas résidente des villes dans lesquelles elle travaille, n'a pas accès aux services publics, en particulier à la sécurité sociale et à l'éducation pour ses enfants, au même titre que les urbains de travailleurs migrants forment aujourd'hu de souche. Celta de la rivière des la majorité de la population dans le delta de la rivière des Perles. À Shenzhen, à la fin de 2012 , on comptait plus de 15 millions de travailleurs migrants pour seulement $3 \mathrm{mil}$ (titulaires du hukou de la lions de résidents permanents (titulaires à étaient 4,8 millions pour une population totale de 12,8 millions $^{12}$.

9. J. De Coster, « Productivity : A Key Strategy of the Hong Kong Textile Outlook International, 68, 1996 Textile p. 96.

10. Federation of Hong Kong Industries, Made in PRD, $2007 \mathrm{http}: / /$ www.industryhk.org/tc chi/fp/fp_res/files/made_in_prd_c.pdf.

11. "Une enquête montre que la population migrante de Shenzhen cép la population locale », op. cit. dépasse 15 millons, Le hukou est un système de résidence instaure à l'époqu maoiste qui séparait strictement les villes et les campagnes et interdisait toute mobilité sociale et géographique. Assoupli depuis les réformes, il reste . discriminatoire.

12. «Guangzhou changzhu renkou 1270.08 wan ren wailai renkou 12. "Guang [ « L population de Canton atteint 12 millions 700 mille, zhan jin sicheng " [ La population de Canton atteint 12 millons la population migrante représente près d'un quart de la population »], http://www.ce.cn/xwzx/gnsz/gdxw/201105/17/t20110517_22424565. shtml.
Lentrée du capital international a donc conduit dans les années 1990-2000 à une " course vers le bas ${ }^{13}$ " affectant les conditions de travail afin de réduire les coûts, avec des conséquences désastreuses pour les travailleurs en termes de non-paiement des salaires, d'accidents du travail et d'absence d'indemnisation en cas d'accident.

Bien que le droit du travail chinois se soit considérablement étoffé depuis les années 1990, avec notamment l'entrée en vigueur de la loi sur le travail en 1995 et de la loi sur les contrats de travail en 2008, l'instauration en 2002 d'un salaire minimum censé être réévalué chaque année en fonction de l'inflation par les autorités locales, ainsi que l'intégration progressive et partielle des travailleurs migrants dans les systèmes de sécurité sociale urbains, son application reste très aléatoire. Cela s'explique par l'absence de droits collectifs (droit de grève, droit de former des syndicats indépendants et de mener des négociations collectives) ainsi que de tribunaux et d'une inspection du travail efficaces et indépendants, dans un contexte de collusion entre les autorités locales et les entrepreneurs ${ }^{14}$.

En 2006, le gouvernement chinois révélait qu'environ 700000 personnes par an restaient paralysées à la suite d'un accident du travail. Aujourd'hui encore, il y a au moins 200 cas d'empoisonnement chaque année, la plupart dans des entreprises hongkongaises, notamment spécialisées dans la fabrication de jouets, situées dans le delta. Beaucoup de victimes ne reçoivent aucune indemnité ${ }^{15}$. De même, les cas de non-paiements de salaire ou d'indemnités en cas de licenciement pour cause de relocalisation des entreprises n'ont cessé d'augmenter ces dernières années avec la baisse des commandes consécutive à la récession mondiale.

13. A. Chan, «Toujours plus bas! Les effets de la mondialisation sur les conditions de travail en Chine », Perspectives chinoises, $\mathrm{n}^{\circ} 75$, 2003 , p. $43-52$.

14. Ch. Froissart, «Les "ONG" de défense des droits des travailleurs migrants. Des organisations proto-syndicales qui contribuent à la stabilité dynamique du régime », Perspectives chinoises, $\mathrm{n}^{\circ} 2,2011$, p. 20-28.

5. "Workers Safety Concerns Linger 20 Years After Factory Fire », http://www.rfa.org/english/news/china/fire-11192013132650.html. 
La montée de l'activisme social : différentes organisations, différentes stratégies

Le développement des liens économiques est allé de pair avec la montée de l'activisme social. De nombreux activistes hongkongais que nous avons rencontrés considèrent en effet que la société hongkongaise a la responsabilité de contrôler les pratiques de ses entreprises en matière d'investissements et de travail en Chine, même si leur action s'étend, bien entendu, à l'ensemble des entreprises étrangères.

Tout a commencé avec l'incendie de l'usine Zhili en 1993. Le 19 novembre 1993 à Shenzhen, un incendie ravage l'usine Zhili gérée par un sous-traitant hongkongais et spécialisée dans la production de jouets pour la multinationale italienne Chicco. L'usine était allée à l'encontre des normes de sécurité en combinant dans un même bâtiment les ateliers de production, les dortoirs des ouvriers et les lieux de stockage. Elle avait également ajouté des barreaux aux fenêtres et condamné les sorties de secours pour prévenir les vols. Résultat : 87 travailleurs, essentiellement des femmes, trouvent la mort ; 47 autres sont gravement brûlés ou blessés. Bien que l'usine soit mise en faillite et le propriétaire emprisonné, les victimes et leurs familles ne reçoivent aucune indemnité. Des organisations d'étudiants, de travailleurs et des organisations caritatives hongkongaises lancent alors une campagne contre la marque à Hong Kong et à Rome, et sont rejoints par les syndicats italiens. Ils mènent parallèlement leur enquête sur les dicatil des ouvriers, se rendent dans les villages pour rencontrer les familles des victimes et lèvent des fonds pour les indemniser. En 1997, Chicco est condamnée à verpour victimes et à leurs familles ${ }^{16}$. D'après les activistes que nous avons rencontrés, cette campagne a également eu un rôle important dans la publication de la première loi chinoise sur le travail en 1995 et les autorités du continent ont depuis renforcé les mesures de sécurité contre les incendies dans les usines, celles-ci étant désormais censées se doter de sorties de secours signalées par un marquage lumineux vert et d'extincteurs à tous les étages ${ }^{17}$.

16. «Jiannan de xunzhao " [« Une quête difficile»], Zhongguo Qingnian bao [Quotidien de la jeunesse], 17 janv. 2001.

17. «Workers Safety Concerns Linger 20 Years After Factory Fire »,
Lun des piliers de cette campagne, l'étudiante Pun Ngai, depuis devenue une activiste célèbre et un professeur reconnu à l'université des sciences et technologies de Hong Kong, fonde en 1996 le China Working Women's Network (CWWN). Cette ONG, enregistrée à Hong Kong mais opérant secrètement à Shenzhen (jusqu'à son interdiction en 2004), a pour objectifs d'informer les travailleuses sur la législation du travail, notamment en matière de sécurité et de santé, mais aussi sur leurs droits en tant que femmes, et de les aider à résoudre des conflits tout en leur apprenant à s'organiser pour promouvoir la démocratie au sein des lieux de travail ${ }^{18}$. L'une des spécificités de cette organisation est qu'elle garde une grande porosité avec les milieux étudiants : les étudiants de Pun Ngai y font souvent des stages, tandis que ses doctorants, suivant son exemple ${ }^{19}$, fondent leurs enquêtes de terrain sur une méthode d'observation bien particulière puisqu'ils se font employer dans les usines chinoises et y travaillent comme ouvriers pendant plusieurs mois.

CWWN a créé une méthode qui a par la suite fait des émules parmi les ONG hongkongaises, comme Labour Education and Service Network (LESN), China Labour Support Network (CLSN), Labour Action China (LAC), Workers Empowerment, fondées au début des années 2000 : celle du « community service » s'appuyant sur une collaboration étroite avec des ONG chinoises situées dans le delta. Ces dernières s'implantent dans les quartiers où se concentrent les usines et les dortoirs des ouvriers, y ouvrant un bureau, assorti parfois d'une petite bibliothèque offrant un lieu de documentation et de sociabilité aux travailleurs, où elles organisent des séances de formation sur le droit du travail et la négociation. Elles interviennent également directement auprès des employeurs en cas de conflit ou aident les

\section{op. cit.}

18. " The Chinese Working Women's Network », http://www. chinadevelopmentbrief.cn/DirectoryofNGOs/?p $=4126$.

19. Le livre de Pun Ngai Made in China. Women Factory Workers in a Global Workplace (Hong Kong University Press, 2005) qui l'a rendue célèbre et a notamment reçu le prix C. Wright Mills, est fondé sur un travail ethnographique de huit mois que l'auteur a conduit en étant employée d'une usine d'électronique à Shenzhen où elle a partagé la vie des ouvrières. 
ouvriers à porter plainte. Certaines de ces ONG tentent également d'organiser les travailleurs en vue d'actions collectives afin qu'ils puissent mieux défendre leurs droits et obtenir de meilleures conditions de vie et de travail. Elles aident à une prise de conscience de ces droits et contribuent à renforcer le pouvoir collectif des travailleurs dans un contexte où les syndicats officiels ne défendent pas les droits des employés mais servent plutôt à les contrôler.

La société civile hongkongaise joue donc un rôle important dans le développement des ONG spécialisées dans la défense des droits des travailleurs dans le delta. D'après nos estimations, environ dix ONG enregistrées à Hong Kong ont des activités en Chine ; il y aurait aujourd'hui une cinquantaine d'ONG chinoises au service des travailleurs dans le delta, dont une bonne douzaine ont un partenariat direct avec des ONG de Hong Kong. Ces organisations chinoises ont souvent vu le jour grâce au soutien des ONG hongkongaises, qui les orientent également dans les stratégies à suivre, les aident à se professionnaliser et à lever des fonds. Cependant, ces partenariats restent fragiles dans la mesure où les ONG du delta sont régulièrement confrontées à la répression des autorités chinoises, qui alternent répression ciblée, harcèlement au quotidien, pression sur les propriétaires pour qu'ils cessent de louer leurs locaux à ces organisations, avec de vastes campagnes d'éradication comme en 2012. Les autorités locales sont souvent de mèche avec les employeurs et ferment les yeux lorsque ceux-ci emploient des hommes de main qui s'attaquent physiquement aux représentants de ces organisations, Qingnan, responsable de l'ONG Dagongzhe Migrant Workers Center à Shenzhen, a été violemment agressé alors qu'il informait les ouvriers sur leurs droits aux abords d'une usine. Bien que l'attaque ait failli lui coûter une jambe, le handicap de Huang a été considéré comme mineur et il n'a pu obtenir qu'une maigre indemnité. Malgré la mobilisation des ONG chinoises, hongkongaises et internationales, et un procès en - appel, le commanditaire et l'exécutant s'en sont tirés avec des peines de prison réduites tandis qu'un deuxième procès en appel a été refusé ${ }^{20}$. Certaines ONG ne se remettent jamais de

20. « Two Years to Fight for a Fair Trial and Verdict for Huang ces attaques mais d'autres tentent de renaître de leurs cendres en déménageant dans un autre district, voire une autre ville du delta, où elles sont alors contraintes de repartir de rien et perdent le contact avec les ouvriers qu'elles avaient formés.

D'autres ONG hongkongaises ont mis en œuvre différentes stratégies : elles se sont spécialisées dans la recherche, la supervision des entreprises et l'« advocacy », dénonçant les conditions de travail inhumaines des sweatshops pour tenter de faire pression sur les multinationales en s'attaquant à leur image, en sensibilisant le public et en tentant d'influer sur les pratiques des consommateurs. Hong Kong est ainsi devenu un haut lieu de la critique avec la création en 2005 de l'organisation Students and Scholars Against Corporate Misbehaviour (SACOM) qui a lancé des campagnes féroces, notamment « Clean-Up Your Computer » en 2006 et « Hi-Tech, No Rights ? » en 2008, accompagnées de nombreux rapports visant en particulier Foxconn, sous-traitant d'Apple, qui ont forcé le secteur de l'électronique à s'ouvrir aux programmes de Responsabilité sociale des entreprises (RSE). LONG a également largement contribué à redéfinir ces programmes, dénonçant les abus et les contournements de la méthode des audits et militant pour une approche «participative » des programmes RSE, grâce à laquelle les ouvriers sont appelés à superviser directement leur entreprise. Un tournant a été pris avec un programme mené chez deux sous-traitants de Hewlett Packard dans le Guangdong en 2009 : les ouvriers ont non seulement été formés au droit du travail mais aussi à élire un comité ouvrier capable de négocier avec la direction de l'usine ${ }^{21}$. Le rôle de SACOM devient alors celui d'un coordinateur : l'organisation propose des ONG chargées de la mise en œuvre du programme (dans le cas du programme HP, LESN et CWWN), en collaboration avec leurs partenaires chinois. Les ONG chinoises s'occupent généralement de la gestion de la hotline qui permet de recueillir directement les doléances des employés et de superviser la mise en œuvre du programme. La coopération entre ONG chinoises et hongkongaises

Qingnan and Shenzhen Worker Centre, China », http://www. workerempowerment.org/en/newsletter/24.

21. "New War of the Flea : CSR and Labor Activism in China ", http://www.china.org.cn/china/2009-10/22/content_18750629.htm. 
s'exerce donc également dans le domaine de la RSE, ces programmes permettant aux organisations locales de transposer le travail qu'elles font au sein des quartiers ouvriers directement dans les usines et de toucher ainsi beaucoup plus de travailleurs. Cependant, l'impact de ces programmes reste généralement limité : s'ils permettent une amélioration des conditions de vie et de travail des ouvriers et apprennent à ces derniers à formuler des demandes collectives auprès de la direction, les sous-traitants n'hésitent pas à revenir sur leurs obligations légales si cela leur permet d'acquérir de nouveaux marchés auprès d'autres multinationales. Par ailleurs, les comités ouvriers disparaissent généralement avec la fin du programme et le pouvoir collectif que ces programmes ont contribué à construire avec la rotation de la main-d'œuvre.

Une autre organisation occupe une place essentielle dans le champ de l'activisme social en faveur des ouvriers chinois : le China Labour Bulletin (CLB). Ancien ouvrier des chemins de fer en Chine dans les années 1980, son fondateur, Han Dongfang, a été le porte-parole du premier syndicat indépendant chinois : la Fédération autonome des travailleurs de Pékin, qui a connu deux semaines d'existence sur la place Tiananmen en 1989 avant que le mouvement démocratique ne soit réprimé. Emprisonné puis libéré pour raisons médicales, Han n'a jamais pu rentrer en Chine après un voyağe aux États-Unis où il était parti se faire soigner. Il décide alors d'utiliser le territoire de la RAS comme base pour poursuivre son combat en faveur des ouvriers chinois et crée CLB en 1994. En prise directe avec la réalité quotidienne des ouvriers grâce à ses deux émissions de radio hebdomadaires - CLB sur Radio Free Asia et Radio Express News - ouvertes aux appels téléphoniques des auditeurs, Han a beaucoup œuvré pour faire connaître la condition ouvrière, notamment l'ampleur des licenciements lors des restructurations des entreprises d'État, la réalité du nombre de victimes des explosions dans les mines de charbon et les stratégies des autorités pour ne pas indemniser les familles, le combat des 6 millions de victimes de la silicose pour faire reconnaître leur maladie et l'évolution du mouvement ouvrier, comme en témoigne le site internet de l'organisation ${ }^{22}$. Laction de CLB est multiple, elle

22. China Labour Bulletin, http://www.clb.org.hk. consiste en particulier à briser l'isolement des ouvriers en favorisant la création d'un réseau de soutien composé d'ONG chinoises et d'avocats, qui les aident à se défendre en justice, les orientent dans leur lutte et favorisent l'émergence de militants et de leaders ouvriers. CLB se concentre depuis quelques années sur des formations aux négociations collectives, organisées dans ses locaux à Hong Kong ou, en Chine populaire, par ses partenaires qui militent également pour que les représentants ouvriers indépendants des syndicats, souvent arrêtés ou licenciés pour leurs activités, obtiennent une protection juridique. Pourtant, Han a progressivement tourné le dos à son engagement premier : la lutte pour la démocratie en Chine et la création de syndicats indépendants ; il croit désormais à la possibilité d'une réforme à l'intérieur du système, notamment des syndicats officiels ${ }^{23}$.

Le delta : un champ de bataille entre ONG et entrepreneurs hongkongais sur la législation du travail

La pression que peut exercer la société hongkongaise de l'autre côté de la frontière reste à double tranchant, comme le montre l'action des industriels de Hong Kong pour contrecarrer le développement de la législation sociale dans le Guangdong. Ces dernières années, l'action conjointe des grèves et des rapports publiés par des ONG comme CLB ou LAC a poussé le Guangdong à préparer un certain nombre de projets de loi très progressistes. Tous cependant ont été mis en échec par les chambres de commerces hongkongaises, qui arguent du fait que ces lois condamneraient à la faillite les PME de la RAS et menacent la Chine de retirer leurs capitaux.

Déjà, lors de la discussion de la Loi (nationale) sur les contrats de travail entre 2005 et 2007, les entrepreneurs hongkongais s'étaient opposé aux deux aspects les plus progressistes de la loi. Lun concernait l'obligation faite aux employeurs de payer une indemnité à leurs employés en cas de licenciement (correspondant à un mois de salaire par année travaillée). L'autre obligeait les employeurs à verser une indemnité équivalant à au moins un an de salaire à tout

23. D.-F. Han (avec la collaboration de M. Sztanke), Mon combat pour les ouvriers chinois, Neuilly-sur-Seine, Michel Lafon, 2014. 
employé quittant l'entreprise en possession d'informations techniques considérées comme secrètes, qui lui interdisaient d'être réemployé dans la zone où se situe l'entreprise pendant les deux années suivant son départ ${ }^{24}$. Bien que le premier point ait également suscité l'opposition des chambres de commerce européennes et américaines, la loi est quand même passée. En revanche, les entrepreneurs hongkongais ont obtenu gain de cause sur les Règlements de la province du Guangdong pour la gestion démocratique des entreprises, les Règlements de la province du Guangdong sur les négociations collectives et les Règlements sur les négociations collectives dans la Zone économique spéciale de Shenzhen rendus publics en août 2010.

Ces trois avant-projets de loi faisaient suite à une série de suicides dans les usines Foxconn ainsi quau vaste mouvement de grèves qui avait embrasé le secteur automobile dans le Guangdong, puis d'autres secteurs sur l'ensemble du territoire, et dont le point de départ avait été la grande grève de 2010 à l'usine Honda Nanhai au cours de laquelle les ouvriers avaient demandé des hausses substantielles de salaire et l'élection des représentants syndicaux. Ces avantprojets constituaient un pas important vers l'institutionnalisation du droit de grève et de véritables négociations collectives, établissant par exemple des procédures de formation de comités de représentation des employés dont les pouvoirs étaient clairement définis. Ils stipulaient par ailleurs que les conseils d'administration des entreprises devaient être formés pour un tiers de représentants ouvriers, permettant ainsi à ces derniers d'avoir une vision claire des bénéfices de l'entreprise et de participer à l'élaboration de ses plans de développement. Un employeur pouvait être contraint à engager une négociation collective sur les salaires si au moins un cinquième des employés le demandait et une consultation sur les salaires devait être organisée au moins une fois par an. Si l'employeur ne répondait pas à cette demande dans les quinze jours et qu'une grève en résultait, il n'était pas autorisé à licencier les grévistes. Enfin, les employeurs ne donnant

24. « Gangshang zhi zhongguo zhiding laodong hetongfa cao'an duochu bu heli » [ "Les entrepreneurs hongkongais pointent que de nombreux points du projet de loi sur les contrats de travail ne sont pas raisonnables »], http://au.epochtimes.com/gb/6/5/15/n1318567.htm. pas suite à ce qui avait été décidé lors des négociations pouvaient être soumis à une amende de 50000 yuans ${ }^{25}$. Jamais la législation n'a été aussi loin, mais jamais non plus l'opposition des entrepreneurs n'avait été si déterminée, ceux-ci ayant même menacé de se rendre à Pékin pour défendre leurs intérêts auprès du ministère du Commerce et du Comité pour la réforme et le développement du Conseil des affaires de l'État. Ainsi mises en échec à l'automne 2010, ces propositions sont depuis restées lettre morte.

Une bataille est donc bien engagée entre Hong Kong et le delta de la rivière des Perles, et par-delà ce dernier, le régime de la République populaire de Chine, comme en témoigne l'ambivalence des flux transfrontaliers. D'une part, les accords du CEPA sont vus par les Hongkongais comme une manière de promouvoir - en créant notamment une dépendance économique de Hong Kong à l'égard de la Chine, incarnée par des flots journaliers de touristes chinois qui suscitent de plus en plus l'hostilité des Hongkongais - une intégration irréversible de la RAS au continent, autrement dit, de favoriser le concept d'" un pays » par rapport à celui de « deux systèmes ». Mais d'autre part, les flux transfrontaliers jouent dans les deux sens et, pour beaucoup d'activistes, ils représentent également une opportunité de faire évoluer les rapports de force au Guangdong et, à travers lui. d'influer sur l'évolution politique de la Chine.

Chloé FroIsSART et Yi XU

25. « Guangdong ni lifa yi gongchao, gongren yi qiguan, gang shang lianshu fandui " [ Le Guangdong étudie la possibilité de légiférer pour restreindre la vague de grèves, les entrepreneurs hongkongais unis dans l'opposition »], http://www.boxun.com/news/ gb/china/2010/09/201009170537.shtml ; Xianggang zongshanghui guanyu "Guangdong sheng qiye minzhu guanli tiaoli ", "Guangdong sheng gongzi jiti tanpan xieshang tiaoli " $j i$ "Shenzhen jingji tequ jiti xieshang tiaoli » de yijian he jianyi » [Opinion et proposition de la chambre de commerce générale de Hong Kong concernant les Règlements de la province du Guangdong pour la gestion démocratique des entreprises, les Règlements de la province du Guangdong sur les négociations collectives et les Règlements sur les négociations collectives dans la Zone économique spéciale de Shenzhen], http://www.chamber.org.hk/ FileUpload/201010061743117980/Democracy\%20Submission.pdf. 